\title{
Pediatría
}

http://www.revistapediatria.org/

DOI: https://10.14295/rp.v54i1.151

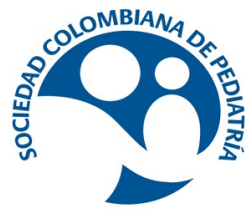

Regional Bogotá

Reportes de caso

\section{Pancreatitis necrosante como presentación atípica de fibrosis quística en paciente preescolar}

\author{
Ricardo Hernández-Sarmiento ${ }^{a}$, Sarah Bastidas-Legarda ${ }^{b}$, Sara Aguilera-Martinez ${ }^{c}$, Maria \\ I. Lozano-Jaramilloc \\ a. Residente de pediatría, Universidad del Rosario, Bogotá DC, Colombia \\ b. Residente de pediatría, Universidad de la Sabana, Bogotá DC, Colombia \\ c. Pediatra, Fundación Cardioinfantil, Bogotá DC, Colombia
}

INFORMACIÓN DEL ARTÍCULO

\section{Historia del artículo:}

Recibido el 31 de julio 2019

Aceptado el 20 agosto 2021

Palabras clave:

Fibrosis quística

Pancreatitis

Sepsis

Pediatría

\section{R E S U M E N}

Antecedentes: La fibrosis quística es una enfermedad de herencia autosómica recesiva, consistente en mutaciones en el gen regulador de la conductibilidad transmembrana, provocando una alteración en la viscosidad de las secreciones. Reporte de caso: Presentamos el caso de una paciente preescolar que ingresa por un cuadro de dolor abdominal crónico en quien se le diagnosticó pancreatitis necrosante como debut de fibrosis quística, quien requirió cuatro meses de hospitalización. Conclusión: La fibrosis quística es una enfermedad con alta tasa de morbimortalidad en la población pediátrica, por lo que se deben conocer sus posibles formas de debut y su clínica, para un manejo oportuno y seguimiento interdisciplinario.

Necrotizing pancreatitis as an atypical presentation of cystic fibrosis in a preschool patient

\section{A B S T R A C T}

Background: Cystic fibrosis is a disease with autosomal recessive inheritance, consisting of mutations in the transmembrane conductivity regulatory gene, causing an alteration in the viscosity of secretions. Case report: We present the case of a preschool patient admitted due to chronic abdominal pain who was diagnosed with necrotizing pancreatitis as a cystic fibrosis debut, requiring four months of hospitalization. Conclusion: Cystic fibrosis is a disease with a high morbidity and mortality rate in the pediatric population; physicians should know its possible debut forms and clinical presentation for timely management and interdisciplinary follow-up.

Keywords:

Cystic fibrosis

Pancreatitis

Sepsis

Pediatrics

\footnotetext{
*Autor para correspondencia. Ricardo Hernández-Sarmiento

Correo electrónico: rhernandezs15@hotmail.com
}

Como Citar: Hernández-Sarmiento R, Bastidas-Legarda S, Aguilera-Martínez S, Lozano-Jaramillo MI. Pancreatitis necrosante como presentación atípica de fibrosis quística en paciente preescolar. Pediatr. 2021;54(1):40-43. 


\section{Introducción}

La fibrosis quística $(\mathrm{FQ})$ es la enfermedad con herencia autosómica recesiva con mayor incidencia en la población caucásica, afectando a uno de cada 2000 a 4000 nacimientos vivos. LA FQ se origina en variantes patogénicas en el gen regulador de la conductibilidad transmembrana (CFTR), provocando una alteración en la viscosidad de las secreciones.

Se considera una enfermedad multiorgánica, ya que incluye afectación pulmonar y pancreática, sin embargo tambien puede producir polipos nasales, azoospermia, hepatopatía crónica, colelitiasis, diabetes mellitus, malnutrición, retraso de crecimiento e ileo meconial en los neonatos (1). En los pacientes con $\mathrm{FQ}$ pueden desarrollarse episodios agudos, aislados o repetidos de pancreatitis, siendo esta última entidad, una posibilidad de debut de la enfermedad. En la literatura, se describe una prevalencia de pancreatitis en la FQ inferior al $2 \%$ (2).

\section{Reporte de caso}

Paciente femenina de tres años previamente sana, con cuadro de un año de evolución de dolor abdominal intermitente que se reagudiza asociado a estreñimiento, distensión abdominal, adinamia, hiporexia, emesis, diaforesis y edema en miembros inferiores. Al examen físico presenta hepatomegalia de $4 \mathrm{~cm}$, onda ascítica positiva. Ecografía abdominal con abundante líquido libre en cavidad. Amilasa en 993 U/L y lipasa en 1504 U/L, por lo que se inició manejo antibiótico con ampicilina- sulbactam. Se realizó tomografía axial computarizada (TAC) de abdomen contrastado y colangioresonancia que documentaron colección del epigastrio compatible con pseudoquiste pancreático de 12 milímetros.

Presentó picos febriles asociado a paraclínicos de control que presentan reactantes de fase aguda positivos, por lo que se diagnosticó sepsis de origen abdominal y se inició manejo antibiótico con piperacilina tazobactam. La resonancia magnética nuclear (RMN) abdominal contrastada, mostró pancreatitis necrotizante mixta mayor al $30 \%$ con presencia de colecciones pancreáticas y peripancreáticas (Figura 1) por lo que se escalona manejo antibiótico a meropenem y vancomicina.

Presentó evolución tórpida, requiriendo múltiples paracentesis evacuatorias, sin respuesta a albúmina, furosemida y espironolactona, por lo que se sospechó fístula pancreática; la cual fue descartada imagenológicamente, además de ocho cultivos de líquido peritoneal negativos y nueve hemocultivos negativos. Ante una paciente con signos de respuesta inflamatoria sistémica y reactantes de fase aguda positivos de forma persistente, se inició cubrimiento antifúngico por sospecha de infección invasiva.

El cuadro clínico se acompañó posteriormente de tos, signos de dificultad respiratoria, requiriendo oxigenoterapia de alto flujo por cánula nasal, con estancia en Unidad de Cuidados Intensivos Pediátricos (UCIP). En la radiografía de tórax se aprecia derrame pleural bilateral con atelectasia del parénquima pulmonar subyacente (Figura 2). La TAC de tórax, evidenció obliteración completa del hemitórax izquierdo (Figura 3), la ecografía torácica indicó una distancia interpleural de 30 milímetros.

Figura 1. RMN abdomen contrastado.

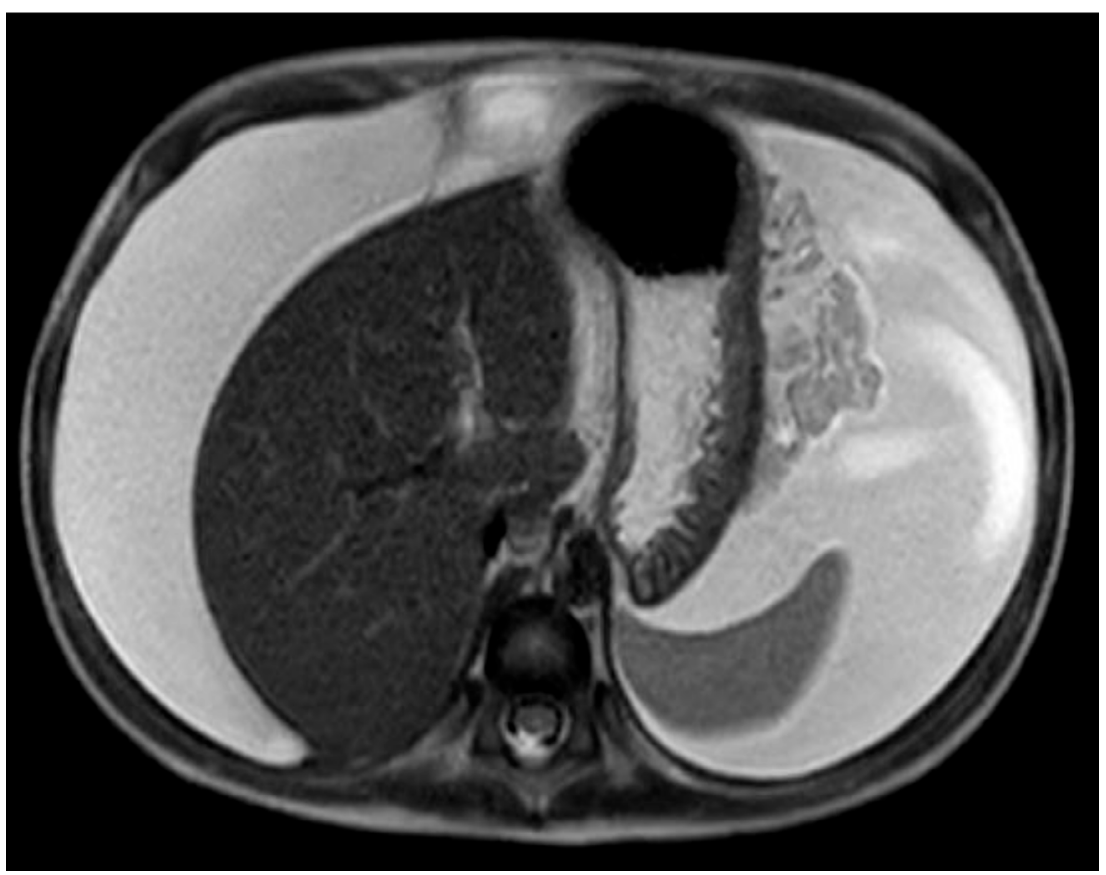

Importante alteración de la morfología del páncreas con defecto de perfusión que compromete más del 30 \% del órgano, asociado a colección pancreática lobulada en la cabeza y gran cantidad de líquido ascítico. 
Figura 2. Radiografía de tórax.

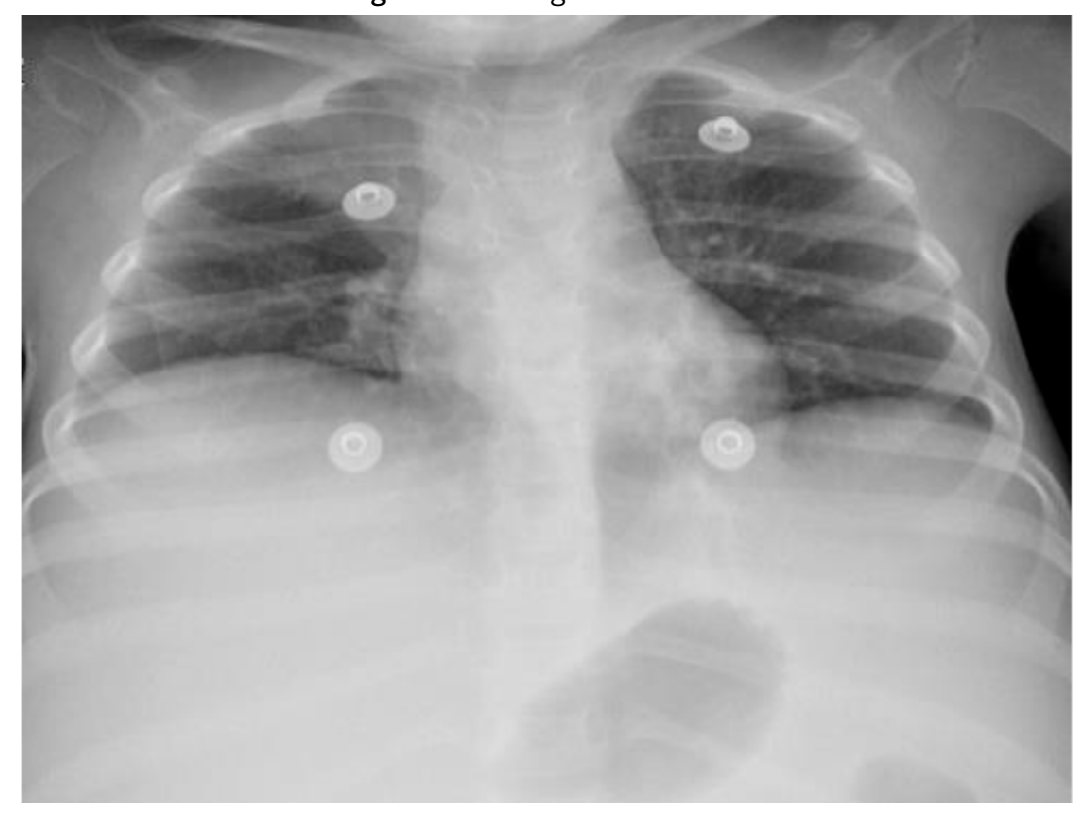

Atelectasia parcial del lóbulo superior derecho.

Figura 3. TAC de tórax.

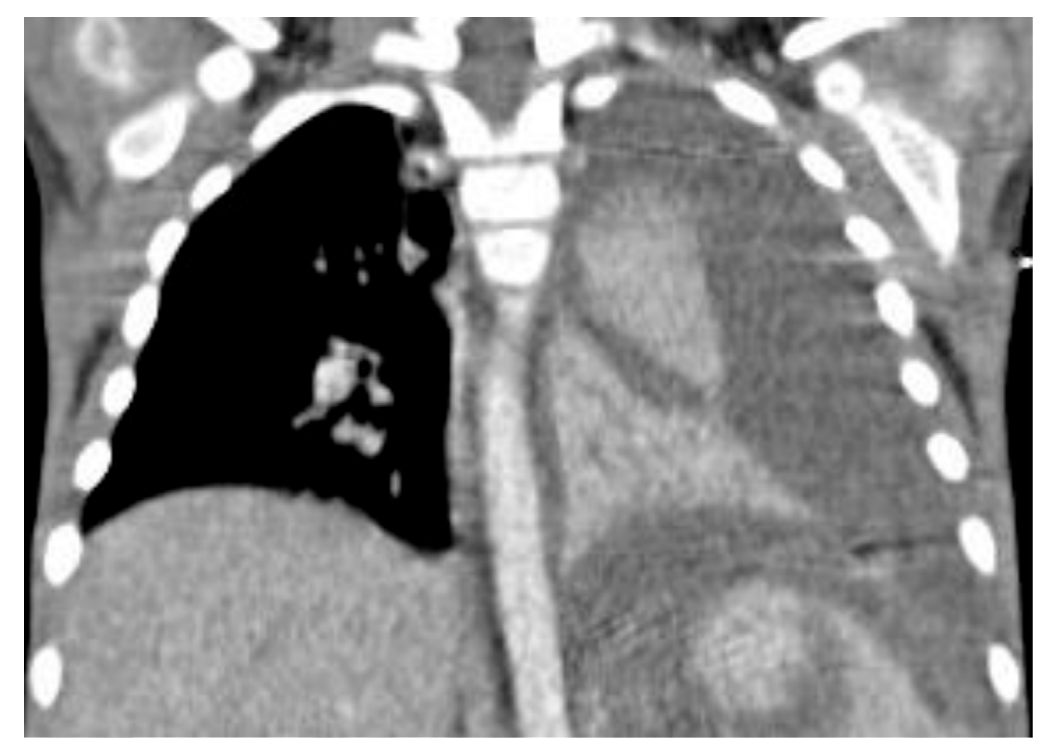

Derrame pleural masivo izquierdo, con líquido en cisura mayor condicionando atelectasia de este pulmón.

Requirió múltiples drenajes de empiema por toracoscopia y toracostomía con aislamiento de Staphylococcus aureus resistente a meticilina. Se realizaron dos electroforesis en sudor por método Nanoduct ${ }^{\circledR}$ que fueron positivas y adicionalmente niveles de IgG4 elevados. Se inició suplencia con enzimas pancreáticas con mejoría del cuadro clínico y se dio egreso a los cuatro meses de hospitalización con órdenes de estudios genéticos para fibrosis quística de manera ambulatoria.

\section{Discusión}

La FQ es una de las enfermedades hereditarias más frecuentes en la raza caucásica, con una frecuencia de portadores sanos de 1/30. Probablemente la primera descripción anatomopatológica se remonta al año 1595, cuando un profesor de botánica y anatomía realizó una autopsia a una niña de 11 años que habia presentado desnutrición y fiebre héctica, encontrando 
un páncreas abultado, cirroso, blanco y brillante, además de signos de pericarditis. Sin embargo, el pediatra Guido Fanconi fue el primero en usar el térmico de fibrosis quística, mientras que la patóloga Dorothy Hansine Andersen describió la enfermedad en 1938, denominándola «fibrosis quística del páncreas» $(1,3)$.

La mutación c.1521_1523delCTT (p.Phe508del) conocida comúnmente como mutación $\triangle F 508$ del gen CFTR (7q31.2), representa más del 60 \% de los enfermos de FQ en el mundo; sin embargo, se conocen más de 2000 mutaciones diferentes que ocasionan esta enfermedad, clasificándose en 6 clases, dependiendo del defecto subyacente; siendo las mutaciones de clase I a III las más graves, asociadas a insuficiencia pancreática con esteatorrea y malabsorción (3).

La FQ también puede conllevar a complicaciones pulmonares incluyendo hemoptisis, neumotórax, aspergilosis, infecciones bacterianas y atelectasias (4). Ésta última, ocurre como resultado de tapones mucosos y enfermedad parenquimatosa severa, las cuales típicamente son tratadas con antibioticoterapia intravenosa y fisioterapia respiratoria, con resultados satisfactorios como en nuestra paciente (5).

Los microorganismos que colonizan la vía aérea de los pacientes con FQ se asocian a la edad del paciente. Durante las primeras etapas de la vida, la infecciones virales provocan denudación del epitelio pulmonar lo cual favorece la colonización bacteriana recurrente y el estado local de inflamación crónica. Tras este periodo inicial, la colonizacion más frecuente es causada por Staphylococcus aureus y Haemophilus influenzae. Conforme avanza la edad del paciente y la progresión de la enfermedad, decrece la colonización por Staphylococcus aureus y aumenta el aislamiento de Pseudomonas aureginosa, hasta convertirse en el microorganismo más frecuente en la edad adulta. Recientemente han aumentado los aislamientos por Staphylococcus aureus resistente a la meticilina (6), tal como se apreció en este caso.

La FQ ya no es la enfermedad clásica que se diagnosticaba en la infancia; cada vez se observan más síntomas heterogéneos leves que conducen a diagnósticos en la edad adulta que suelen debutar como enfermedad de un solo órgano o multisistémica leve. La afectación pancreática es más común y la afectación pulmonar suele ser leve (2).

En el páncreas, la proteína CFTR se expresa en la membrana apical de las células ductales proximales y su alteración en la FQ se traduce en una disminución de la secreción ductal de cloro con la consiguiente falta de activación del intercambio cloro-bicarbonato, con persistencia de producción enzimática por parte de las células acinares y una insuficiencia pancreática exocrina como resultado (7).

La pancreatitis es una complicación tardía de la FQ con una prevalencia estimada entre el 0.5 al $1 \%$ en la mayoría de las series publicadas. Se presenta en su mayoría en la adolescencia y en la edad adulta (8). Sin embargo, en un estudio realizado por Sojo y cols, con una muestra de 520 pacientes con diagnóstico de $\mathrm{FQ}$, se presentaron 17 casos de pancreatitis (3.3\%), revelando una prevalencia mayor a las encontradas en la literatura (2). A pesar de que la pancreatitis aguda en la infancia se ha incrementado de forma progresiva en los últimos años, aún no se conoce con exactitud su incidencia, ya que solo existen pequeñas series de casos (9). Aunque el manejo de la pan- creatitis aguda secundaria a la FQ no difiere del aplicado en las pancreatitis de otras etiologías, es importante el diagnóstico etiológico para la posibilidad de realizar consejo genético (10).

En el presente caso, el enfoque clínico fue complejo desde su ingreso, ya que la paciente presentaba signos y síntomas inespecíficos y luego de múltiples procedimientos no se apreciaron característicos, requiriendo varias juntas multidisciplinares en las cuales no se logró determinar la causa de pancreatitis necrosante que la condujo a sepsis de origen abdominal y a falla respiratoria restrictiva.

Dados los picos febriles, se inició cubrimiento antibiótico, el cual se escalonó según recomendación de infectología pediátrica por la evolución tórpida de la paciente. Posterior al tratamiento específico de la FQ tras el diagnóstico, la paciente obtuvo una notable mejoría clínica y paraclínica, motivo por el cual se consideró podía continuar seguimiento de manera ambulatoria. Por lo anterior, es menester considerar la FQ como diagnóstico diferencial en pacientes que presenten pancreatitis para poder realizar un manejo oportuno.

\section{Conclusiones}

La FQ es una enfermedad con alta tasa de morbimortalidad en la población pediátrica, por lo que se deben conocer sus posibles formas de debut y su clínica, para un manejo oportuno y seguimiento interdisciplinario.

\section{R E F E R E N C I A S}

1. Navarro S. Recopilación histórica de la fibrosis quística. Gastroenterol Hepatol. 2015.

2. Sojo A, Martínez N, Bousoño C, García M, Heredia S, Mazanares J, et al. Pancreatitis en la fibrosis quística: correlación con el genotipo y estado pancreático. An Pedatr. 2011;75(6):401-8.

3. Santana E, Tamayo V, Collazo T, López I, Feria F, Rodríguez F. Caracterización clínica y genética de la fibrosis quística en la provincia de Holguín. Rev Cuba Pediatr. 2017;89(2):136-44.

4. Restrepo-Gualteros S, Milena S, Muñoz A, Quevedo J. Complicaciones pulmonares en fibrosis quística. Reper Med Cir. 2016;25(1):22-32.

5. Delgado I, Moreno M, Carrasco L, Marín L, Muñoz P, Moreno M, et al. Atelectasia persistente en paciente con fibrosis quística: ¿debemos tratarla siempre con antibioterapia? Arch Bronconeumol. 2019;55(1):54-5.

6. Ruiz de Valbuena M. Fibrosis quística y sus manifestaciones respiratorias. Pediatr Integr. 2016;XX(2):119-27.

7. Guarner L. Fisiopatología, diagnóstico y tratamiento de la insuficiencia pancreática exocrina en el paciente con fibrosis quística. Gastroenterol Hepatol. 2005;28(Supl 2):29-32.

8. Ledesma S, Álvarez A, Torres M, de Gracia J. Pancreatitis aguda como forma de presentación de una fibrosis quística. Med Clin. 2003;120(10):399.

9. Villamañán A, de Vicente C, García R. Pancreatitis aguda secundaria a Campylobacter jejuni en un niño afecto de fibrosis quística. Med Clin. 2017.

10. Foruny J, Moreira V, Maiz L, Carrera E, Casals T. Pancreatitis aguda recidivante como forma de presentación de fibrosis quística. Gastroenterol Hepatol. 2005;28(1):20-2. 